\title{
Operating Analysis and Data Mining System for Power Grid Dispatching
}

\author{
Haiming Zhou ${ }^{1}$, Dunnan Liu' ${ }^{2}$, Dan $\mathrm{Li}^{1}$, Guanghui Shao ${ }^{3}$, Qun $\mathrm{Li}^{3}$ \\ ${ }^{1}$ China Electric Power Research Institute, Beijing, China \\ ${ }^{2}$ School of Economics and Management, North China Electric Power University \\ ${ }^{3}$ Northeast China Grid Company, Shenyang, China \\ Email: haimingzhou@163.com, liudunnan@163.com, lidan@163.com
}

Received January, 2013

\begin{abstract}
The dispatching center of power-grid companies is also the data center of the power grid where gathers great amount of operating information. The valuable information contained in these data means a lot for power grid operating management, but at present there is no special method for the management of operating data resource. This paper introduces the operating analysis and data mining system for power grid dispatching. The technique of data warehousing online analytical processing has been used to manage and analysis the great capacity of data. This analysis system is based on the real-time data of the power grid to dig out the potential rule of the power grid operating. This system also provides a research platform for the dispatchers, help to improve the JIT (Just in Time) management of power system.
\end{abstract}

Keywords: Power Grid Dispatch; Index System; Data Mining

\section{Introduction}

Network control center is the center of the grid operating data, a large amount of data that produced from SCADA, EMS, CPS and other systems contains a grid in the operation and safety of all of the information. However, the current dispatch center information system is mainly for data collection, storage and simple summary of query of daily operations, and not specifically for a lot of historical information for effective category management and correlation analysis, cannot effectively use the data from the valuable resource the data extract useful knowledge paired scheduling management.

Data mining techniques [1] is a large database from extracting previously unknown information about the operability of the knowledge discovery process, including data warehouse [2], online analytical processing OLAP [3], and data visualization of the new information science technology. Data mining is widely used in aviation, finance, telecommunications and other areas, which have greatly improved data management and decision-making; in the power industry, data mining system has been in power market analysis, supply market analysis and other fields to be effectively applied. Therefore, it is technologically advanced and practical feasibly for data mining techniques applied to power scheduling analysis.

The measures of establishing dispatching operation analysis and data mining systems, effectively manage- ment and scientific analysis for power grid data, do good use for the summed experience of power grid, the exploration of the power grid law in order to provide the analysis platform for the dispatchers and improve the level of dispatching and scientific decision-making.

This paper first describes the content of the dispatching operation analysis and the system of the index evaluation, and then describes the data warehouse platform and the analysis techniques of multidimensional data of data analysis, finally describes examples of data mining with the actual needs of dispatching operation.

\section{Dispatching Content}

It is supposed to monitor and compute the status of all aspects of dispatching operation, in order to facilitate the refinement of grid operation management, accumulate data and experience under the conditions of the electricity market, continuously improve network security, quality, economic operation, and ensure grid security and stability.

Dispatching indicator system is to quantitatively reflect the operations of all aspects of the grid, and make comprehensive and clear understanding on the main features of network parameters for the scheduling staff through the calculation and analysis of a series of core indicators.

According to State Grid Corporation of scheduling system [4] requirements, grid dispatching departments 
need to analyze and evaluate the power grid control class, scheduling classes, statistical analysis class, with the purpose of forming the closed-loop feedback mechanism of the accuracy of network control and the rationally scientific operation mode, and to achieve meticulous management of the power grid.

\subsection{Run Control Class Indicators}

Categories grid operation control targets should real-time record automatically generated indicators report and power grid index quantification statistics according to the EMS and other automated systems for the inspection of the relevant analysis.

Scheduling on duty should be able to real-time view the running control class indicators and the basic content, in order to adopt appropriate control measures to continuously improve the level of the grid control. These include the following indicators:

1) Frequency indicators:

Include the highest power, lowest frequency, out of 50 $\pm 0.2 \mathrm{~Hz}$ of the frequency of the more limited running time, $50 \pm 0.1 \mathrm{~Hz}$ of frequency pass rate.

2) Tie-line indicator:

Including the tie-line A1/A2 or CPS1/CPS2 more limited number.

3) Important trends of bound cross-section:

Include the cumulative time and the percentage of total calendar time of an important trend over stable section running limits, the important trend of cross-section limit of $90 \%$ to $100 \%$ of the total time and percentage of total calendar time.

4) Standby indicator:

Include: real-time spinning reserve capacity and day 96 load ratio of the percentage of the maximum and minimum values, the ratio of the percentage of an FM unit Number of units and operating units Number of units, the total available capacity of investment in an FM unit, minimum spinning reserve capacity during peak hours, spinning down maximum output capacity during low hours.

5) Voltage indicators:

Include the voltage passing rate of the plant site under the jurisdiction, the proportion of the total number of stations under the jurisdiction of plants of the lower limit of the peak voltage, the proportion of the number of jurisdiction of plants under the total number of stations in the low hours of maximum voltage.

\subsection{Scheduling Class Indicators}

1) Load characteristics of indicators:

Include: maximum / minimum load, maximum load at the same time rate, which is the whole network maximum load and net provincial, city (region or control area) ratio of the sum of the maximum load; net provincial, city (region or control area), maximum rate of change on rise and decrease of 96 points load in the more than 220 $\mathrm{kV}$ substation, the deviates of 96 points on the load forecast and the actual load value more than $\pm 2 \%$ of the points and so on in net provinces (or control area).

2) Power balance indicators

Include: the completion of day, month power supply; the completion of selected power plant project.

3) Standby indicator:

Include: spinning reserve to meet the rules of order, 10 minutes to bring up the emergency and alternative situation, 30 minutes to control the load standby conditions.

4) Maintenance plan targets:

Include: the completion of scheduled maintenance and the rationality of repairing plan.

5) Indicators of low-frequency load shedding:

Include: real cut capacity of low-frequency load shedding partition, the real fault cut capacity to meet the various regions of the maximum possible N-1 components.

6) System automatic safety device index:

Include: the adaptability of installation strategies, the rationality of device configuration.

7) Voltage indicators:

Include: the rationality of the central point reactive power arrangement, the average in the plant station under the jurisdiction of the peak voltage, low hours.

8) Important indicators of the transmission section:

Include: the number and total time of the running trend over stable limit, the number and total time of the running trend in the $80 \%$ to $100 \%$.

\subsection{Use of KPI}

Once we've identified the Key Performance Indices for the business, it is ready to filter these down to the analyzers. The KPI usually have these three functions:

1) Reflect the Targets

Where possible offer incentives linked to targets, and encourage analyzers to involve staff in the setting of their targets. For example, a sales analyzer may set the sales team targets of a certain number of new contacts per week or visits per month. A production manager might set targets for output, reject rate or work breaks.

2) Find the Cause

Set review dates and compare against previous figures, your business plan, budget or other agreed standard. If you spot an anomaly or problem, the KPI will help to backtrack and pinpoint the cause.

3) Report regularly

Communicate performance figures regularly. Keeping employees updated will encourage them to focus on meeting or exceeding their KPI. 


\subsection{Statistical Analysis of Categories of Indicators}

Statistical analysis indicator refers to the statistical analysis of the previous two categories of indicators during a period of time.

1) Total statistics:

The purpose is that to stat the cumulative quantity of some indicators over a period of time. Such as: failure time (seconds) of frequency, the unqualified cumulative number of days and failure time, the maintenance ticket number and the operating ticket number performed monthly, power grid network losses, unplanned generator outage times, and the frequency of fault trip of power lines, bus, together (main).

2) Mean / extreme value statistics:

The purpose is that to stat the maximum, the minimum or the average indicators over a period of time.

Such as: the largest number of operating ticket in single-day, the 96-points load characteristic curve, together (main) maximum load change rate, water consumption rate of direct transfer hydropower plants, thermal power plant coal consumption rate of direct transfer, high- voltage transmission network net loss, $220 \mathrm{kV}$ and more rapid protection line fault removal rate, times of power cuts and the average daily capacity and so on.

3) Percentage statistics:

The purpose is that to stat the ratio between the two types of indicators of the total in a period of time. Such as: the correct rate of the moves of security control device, the tie-line bias and frequency control passing rate, voltage passing rate, load forecasting accuracy, the passing rate of the low-frequency load shedding control capacity, the operation rate of unit PSS, the operation rate of unit AGC, the qualified rate of the operating vote.

\section{Scheduling Operaion Analysis Data Warehouse Platform}

\subsection{Scheduling Integrated Data Warehouse Platform}

All the available data relative to the supplier's bidding can be divided into three classes:

Data Hub is an online system that is designed for Stats analysis and decision support applications. It could meet the decision support and online analytical applications require all. This data is called data warehouse platform.

The establishment of dispatching and comprehensive analysis platform for data mining is responsible for collecting all kinds of the required scheduling run data indicators in regular time.

1) The basic information of network parameters, power plants and units.

2) Power generation, by the power, the load plan and actual data.
3) Power line trend data, the node voltage data.

4) Local power plants planned and actual data.

5) AGC unit and various indicators of data assessment.

6) Market transactions and load forecast data.

7) Reporting of transactions, transaction data.

8) All kinds of information in regional power market.

9) Regional power grid parameters and inter-provincial trade data.

Data warehouse is a new application of a database technology, and so far, the data warehouse is a relational database management system to manage the data.

\section{Olap Multidimensional Data Analysis and Reporting System}

Data warehouse contains a lot of data extracted from a number of databases, but these data could play its proper value only by using the right tools and being used effectively.

The methods of data mining for the data in the data warehouse to be analyzed include online analytical processing (OLAP), association rule mining, decision tree analysis, cluster analysis and other. Shanghai power system mainly uses online analytical processing (OLAP) technology.

\subsection{Dispatching Multi-dimensional Analysis and Processing}

Multi-dimensional data extraction and OLAP data analysis could be done by the use of all kinds of data in the dispatching data warehouse. As shown in Figure 1:

1) Time dimension: Data classification according to year, quarter, month, week, day, hour and minute.

2) Period dimension: Data classification according to the three period-peak, trough, and waist load.

3) Regional dimension: Data classification according to different regions.

4) Plant dimensions: Data classification according to power plant, the type (bid/peaking/FM/self) and Power Generation Group.

5) Unit dimensions: Data classification depending on the capacity of the unit.

6) Line dimensions: data analysis depending on the line, the line type.

7) Substation dimensions: Data classification according to the type of substation and substation.

8) Weather dimensions: Data classification according to the temperature, humidity, the sunny weather or the cloudy weather and other standard.

9) Custom dimension: We could create new data classification flexibly according to the needs of scheduling Analysis, such as the level of the plant load factor, power frequency, peak and valley levels and any other levels of parameters. 


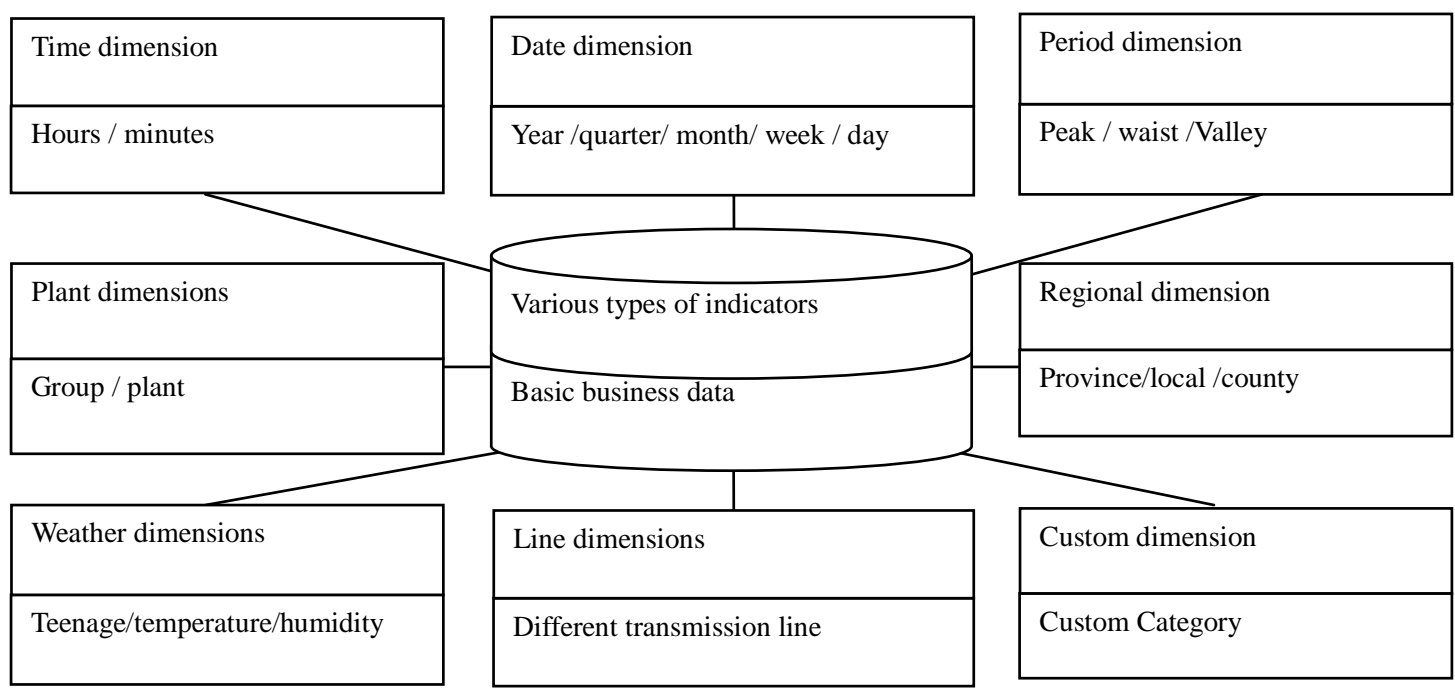

Figure 1. Multi-dimension analysis for power grid data.

\subsection{OLAP-based Reporting System}

The previous power system analysis is based on a variety of control model calculations in advance of the simulation analysis, the schedule data warehouse platform established by the project, aims to statistically analyze and data excavate a large number of historical data, and find the potential operational law from the long-term accumulation. These all cannot be achieved in any previous analysis methods and models.

Power grid analysis is divided into day, monthly and yearly analysis, in accordance with the Requirements of refinement of grid operation. Scheduling classes, statistical analysis of categories of indicators should be months, years for analysis. The reporting system should be based on real-time automation systems such as EMS automatic generation of indicators recorded statements and quantify the power grid index statistics for the inspection of the relevant power system analysis. Dispatchers on duty should be able to view real-time basic and operational control content class indicators, in order to adopt appropriate control measures to continuously improve the level of the grid control.

1) Day analysis: The aim is to find grid operation control problems and deviations of yesterday, and find out the reasons, and to remedy the problems in the next day's operation, to continuously improve the level of operational control.

2) Monthly analysis: Mainly through the analysis of the type of operation plan, statistical analysis class indicators to verify the adaptability of operating mode on the month, in-depth study of the problems and deviations, and improve it in the second month operation mode arrangements, at the same time, to make recommendations and comments for other departments and continuously improve the scheduling refinement level.
3) Annual analysis: The aim is to make suggestions and comments for company planning and technical innovation mainly through the analysis of statistical analysis indicators combined with annual operational mode. Then it provides support for the operational mode in the next year.

The operator is supposed to strengthen communication and coordination with the company development of infrastructure, production, safety supervision, marketing, trading and other relevant departments, and implement corrective measures according to the important issues reflected in the power grid power system analysis.

The purpose is to get the patterns and trends related to grid operation through the power grid power system analysis statistics and analysis, as the power grid summary information and to make decision thought on the future operation mode of preparation and decision.

\section{Dispatching Data Mining Analysis System}

\subsection{Dispatching Data Mining Analysis Methods}

Data Mining (Data Mining) is to extract the implicit in the work, people do not know in advance, but is potentially useful information and knowledge from a large number of, incomplete, noisy, fuzzy, random data.

The methods and procedures of data mining for the data of grid dispatching are as follows:

1) Statistical analysis of indicators

It can create various indicators to analyze according to the needs of data analysis, including:

a) Indicators statistics: Aggregate value, maximum / minimum / average, expectations, variance, standard deviation.

b) Indicators score and early warning: You can score all types of indicators, based on operating experience, 
determine early warning threshold on the basis of statistical indicators.

2) Multivariate correlation analysis

For any amount of view two or more groups, you can associate the following methods analyzed in order to study the association and mutual influence:

a) Qualitative analysis: You can observe the changes of the curve and relevance by the use of data visualization technology, the multiple dimensions of data according to different categories, in 2D or 3D graphics on display, rotation, and perspective.

b) Quantitative analysis: Relevant statistics, analysis and factors affecting the amount of view between the correlation coefficient, covariance.

3) Other analytical methods

Data mining itself reflects a thinking that with the appropriate mathematical methods to explore the law from the data. Data mining does not require one or a few methods to analyze data. In contrast, data mining method broadly define to include all kinds of statistics, signal processing, information science, computer graphics, artificial intelligence and other areas of different methods, depending on the data content, you can use different methods.

Commonly used methods include: cluster analysis, classification, prediction, regression, power system analysis the evolution, correlation analysis, correlation analysis and other methods.

\subsection{The Practical Application of Dispatching Data Mining}

Power dispatching data mining can be applied to the following practical issues.

1) The analysis of the reasonableness of the electric power grid power system.

Analysis methods: analysis of local power generation planning and load correlation, analyze tie-line planning and load correlation.

2) Analysis of electricity contract in real-time

Analytical purposes: to ensure the fairness of the power plant contract implementation and scheduling.

Analysis methods: monitoring all electricity plants contract in real-time completion, monitoring all power plants project plan and the actual deviation; by Dispatchers three shifts and each team for the electricity comple- tion of sequencing analysis.

3) Analysis of various factors in CPS

Purpose of analysis: analyze various factors on the impact of CPS indicators, to improve scheduling policy.

Analysis methods: To list CPS-related factors, to stat the correlation between the factors and CPS indicators by the time dimension, to sort the factors relation, and to give scheduling control recommendations to improve CPS.

\section{Conclusions}

Dispatching operational analysis and data mining system described in this paper is the implementation and enforcement to the schedule of dispatching operational analysis. The calculation and analysis of the dispatching indicators gives the power grid dispatchers a more comprehensive, clear, quantitative understanding. The data mining of the historic data can facilitate the understanding of some complex related issues in the dispatching operation. The author hopes that this research practice could make contributions to the work of dispatching operational analysis.

\section{REFERENCES}

[1] J. Q. Zhao, S. Yan, X. Xiao and Y. Z. Zhou, "Design and Implementation of Double-core Redundant Power Grid Dispatch Automation System," Automation of Electric Power Systems, Vol. 33, No. 21, 2009, pp. 101-103.

[2] S. M. Wang, S. N. Wu, D. L. Zhou and W. C. Wu, "Research on Dispatch Training Base Construction Scheme for Jiangxi Power Grid,” Electric Power, Vol. 42, No. 4, 2009, pp. 70-74.

[3] “ISO Market Monitoring \& Information Protocol," California Independent System Operator Corporation FERC Electric Tariff First Replacement, Vol. 2, No. 497.

[4] H. L. Jin and H. Liu, "Research on visualization techniques in data mining," Proceedings of the 2009 International Conference on Computational Intelligence and Software Engineering, 2009, p. 3.

[5] A. Koretsune, S. Aoki, T. Konzo, H. Tsuji, S. Shimano and E. Mimura, "DEA-based data mining for energy consumption," 10th IEEE International Conference on Emerging Technologies and Factory Automation, Vol. 1, 2005, p. 4.

doi:10.1109/ETFA.2005.1612646 